\title{
An Examination of Transition Models and Processes: Introduction of an Integrated Approach
}

\author{
Kennedy Musamali, EdD \\ Student Support Services, Wichita State \\ University, Wichita, Kansas, USA
}

\begin{abstract}
A transition is the process initiated in response to a change event. It is characterized as an adaptive response to change over time marked by periods of disruption and distress. Many researchers have proposed models to facilitate transition management and successful adaptation. However, a review of the literature revealed while there were multiple transition models, many were limited in their scope and few offered a comprehensive approach to transition management. Furthermore, although studies revealed transitions were essential to successful adaptation, few studies examined transition as a central concept in normative change and adaptive processes. This paper reviews transition models and proposes an integrated framework with a comprehensive approach to transition management.
\end{abstract}

Keywords: transition model, normative change, adaptive experiences

\section{INTRODUCTION}

Many researchers have confirmed that adaptation to change is influenced by the transition process (Brammer \& Abrego, 1981; Bridges, 2003; Bussolari \& Goodell, 2006; Kralik, Visentin, \& Van Loon, 2006; Nicholson, 1990; Schlossberg, Waters, \& Goodman, 1995). A transition is an adaptive response to change that is punctuated by periods of uncertainty (Golan (1983; Kralik et al. 2006). It is described as the process of moving from a comfortable and familiar state to a new or unaccustomed situation (Janusz \& Walkiewicz, 2018). Although transitions provide a framework in which experiences of coping with change can be described and addressed, it is argued that unmanaged transitions make adapting to change challenging and difficult (Bridges, 2003; Meleis, 2015). In other words, "It isn't the changes that do you in, it's the transitions" (Bridges, 2003, p. 3).

While an analysis of the literature supported Bridges assertion, a limited number of studies examined transition as a central concept in managing normative change events (Bridges, 2003, Nicholson, 1990; Schlossberg et al., 1995). Rather, most studies examined the role of transitions in pathology and traumatic health related recovery processes (Bussolari \& Goodell, 2006; Meleis, 2010; Miller, 2010). Specifically, a literature review conducted by Kralik et al., (2006) revealed that transition was a central concept in more health-focused scholarly journals compared to journals with a social focus. In other words, while transition was considered essential to successful adaptation outcomes, it was infrequently examined as a central issue in the management of normative change and adaptive experiences.

Additionally, the literature revealed that while there were multiple transition models, many were limited in their scope and few offered a comprehensive approach to transition management. For instance, Schlossberg's (1981) transition model focused on adults in transition and the assessment of coping resources. Similarly, Nicholson's model examined causes, trends, and challenges of occupational transitions (Nicholson, 1990; Schlossberg et al., 
1995). Likewise, Bridges' (2003) model focused on life transitions and presented a sequential linear framework that did not address the chaotic and unpredictable nature of the transition process. While Bussolari and Goddell's (2006) model addressed the chaotic and unpredictable nature of transitions, it did not provide a clear structure or systematic process of navigating through the unstructured and chaotic nature of a transition process.

Consequently, the purpose of this paper is to examine transition models and propose an integrated framework that offers a more comprehensive approach to transition management. The proposed Kennedy's Integrated Transition (KIT) model is based on the notion that transitions are constructive and transformative cycles. Therefore, deconstruction and reconstruction processes are presented as key phases of the transition cycle (Freedman \& Combs, 1996; McKenzie \& Monk, 1997).

More importantly, the KIT's framework expands on the scope of past models and offers a more comprehensive approach to transition management (Berger \& Luckmann, 1966; Rosen \& Kuehlwein, 1996). For instance, the model's framework explains both normative and unique transitional experiences. Furthermore, the transition activities of disruption, reorganization, integration, and internalization are discussed (Bridges, 1980; Bussolari \& Goodell, 2006; Kralik et al., 2006). Specifically, the impact of transition activities on routines, roles, and relationships are examined (Schlossberg et al., 1995). In contrast to past models, the levels of intensity and role of disruptive activities in transition management are discussed. The paper begins by providing an overview to facilitate an understanding of transitions and change events.

\section{Understanding Transitions}

\section{LITERATURE REVIEW}

According to Bridges (2003), “It isn't the changes that do you in, it's the transitions" (p. 3). A transition is defined as a period or process of changing from one state or condition to another (https://en.oxforddictionaries.com/definition/transition). Specifically, the word derives from a Latin word transire or transition, which means, "to go across." Kralik et al. (2006) characterized transition as a convoluted period that followed a disruptive life event. Similarly, Scholssberg (1981) defined transition as a process triggered by a change event. While Bridges (2003) characterized transition as an internal psychological process triggered by an external change event, Bussolari and Goodell (2009) described transitions as chaotic and disruptive periods that initiated a reevaluation of one's assumptions and behavior. In summation, a transition is an adaptive process initiated in response to a change event (Bridges, 1980; Kralik et al., 2006, Schlossberg, 1981). However, transition processes are characterized by disruption and transition management is essential to facilitate successful adaptation to new situations (Bussolari \& Goodell, 2009).

Importantly, change events trigger disruptions that are distressing to routines, roles, and relationships (Bussolari \& Goodell, 2009; Schlossberg et al., 1995). Therefore, managing transitions requires taking care of disrupted routines, roles, and relationships. Routines are defined as uninterrupted and unproblematic operating procedures that maintain our internalized realities and sense of self (Berger \& Lackmann, 1966). Similarly, role refers to a sense of self that allows an individual to engage his or her surroundings in a meaningful way, while a relationship is the connection one has with people (Oatley, 1990). Consequently, managing disrupted routines, roles, and relationships is essential to successful transition outcomes (Bridges, 2003; Bussolari \& Goodell, 2009; Schlossberg et al., 1995).

Specifically, disruptions are interruptions that render an individual's usual problem-solving mechanisms ineffective (Brammer \& Abrego, 1981, Schlossberg, 1981, Schlossberg et al., 
1995). More importantly, disruptions render familiar assumptions and behaviors ineffective in new situations. This experience leads to feelings of discomfort, anxiety, and helplessness (Moo \& Tsu, 1976). Although transitions require a change in assumptions and behaviors to facilitate adaptation to new routines, roles, and relationships (Schlossberg et al., 1995), a successful transition results in feelings of contentment and mastery over disruptions (Kralik et al., 2006).

In summary, transitions disrupt established routines, roles, and relationships, and prompt the need to reconstruct a valued sense of self (Bussolari \& Goodell, 2009; Kralik et al., 2006; Schlossberg et al., 1995). Specifically, transitions are periods of adjustment and adaptation to new situations (Brammer \& Abrego, 1981; Bussolari \& Goodell, 2009; Nicholson, 1990). Ultimately, a successful transition is characterized by a sense of restored equilibrium, feeling of contentment, and mastery over a disruptive change event (Bridges, 2001; Bussolari \& Goodell, 2009; Nicholson, 1990; Schlossberg, 1981).

\section{Change events}

According to Schlossberg et al. (1995), transitions are triggered by anticipated, unanticipated, or a non-event. Anticipated events are expected occurrences such as marriage, birth, first job, and retirement, while unanticipated events are unexpected and unscheduled occurrences. Unanticipated events include illness, death, divorce, or unplanned unemployment. Non-events are expected events that did not occur and may include marriage or promotion. These change events or non-events trigger transition cycles that carry a sense of loss even when the change is positive or planned (Bussolari \& Goodell, 2009). More importantly, the separation with ones known past elicits a sense of loss with familiar experiences and expectations while the threat of an unknown future activates negative emotions of fear and anxiety (Bussolari \& Goodell, 2009). Specifically, change events elicit transition cycles that are marked by periods of disruption and distress (Bridges, 2001; Bussolari \& Goodell, 2009; Schlossberg et al., 1995). Most importantly, transition cycles are characterized by processes of adjustment and adaptation (Brammer \& Abrego, 1981, Nicholson, 1990; Kralik et al., 2006).

\section{The Transition Cycle}

Transition cycles begin with an end to a familiar status quo and conclude with the adaptation to a new situation (Bridges, 2001). Disengagement from established routines and separation from a familiar context characterize the initial phase of a transition cycle (Bridges, 2001; Schlossberg, 1995). This is followed by an adjustment process initiated to accommodate demands of new routines and expectations of a new situation. Transition cycles end with the incorporation of new practices and an adaptation to a new situation (Bridges, 2001; Bussolari \& Goodell, 2009; Kralik et al., 2006; Schlossberg et al., 1995). Consequently, transition cycles are periods of adjustment and adaptation (Bridges, 2003; Nicholson, 1990).

\section{Adjustments and adaptation}

Adjustments are temporary measures taken to manage disruptions triggered by a change event (Bussolari \& Goodell, 2009). Specifically, disruptions initiate discomfort and distress that prompts the need for cognitive, emotional, and behavioral adjustments. According to Kralik et al. (2006), the discomfort and distress experienced in transitions signal a need for adjustments in psychological and behavioral processes. Bridges (2001) described adjustments as modifications initiated to alleviate distress and manage disorganization generated by the disruptions of a change event. Consequently, reorganization was essential in managing transitions (Bridges, 1980; Levine, 1976). According to Bridges (2003), the process of reorganization included reevaluating and modifying one's assumptions and behaviors to meet the demands of a new situation. However, while adjustments were necessary to stabilize a 
transition process, the main goal of a transition was adaptation (Bridges, 2001; Bussolari \& Goodell, 2009; Kralik et al., 2006; Schlossberg, 1981).

Adaptation occurs when newly acquired assumptions and behaviors are fully integrated and internalized (Bridges, 1980; Bussolari \& Goodell, 2009). In other words, adaptation occurs when newly acquired assumptions and behaviors are accepted and habituated. This process is completed when cognitive, affect, and behavioral activities are aligned and synchronized with the realities of a new situation (Bridges, 2003; Kralik et al., 2006). Adaptation engages a system of coping process that are designed to restore and maintain one's equilibrium (Senesac \& Roy, 2015). Therefore, adaptation is a transformative process that helps restore equilibrium and a lasting sense of stability following a change event (Bussolari \& Goodell, 2009). In sum, processes of adjustment and adaptation mediate successful transitions (Bussolari \& Goodell, 2009; Schlossberg, 1981).

\section{A review of transition models: Conceptual framework and processes}

Transition models provide a framework that allows the conceptualization of transition processes. According to Schlossberg et al. (1995), transition models are conceptualized and supported by contextual, developmental, lifespan, and transition theoretical framework. A contextual framework envisions transitions through a social context, while the developmental theoretical approach views transition through a sequential adult development process (Schlossberg et al., 1995). Specifically, the contextual framework explains the transition process utilizing established social, economic, and cultural practices. In contrast, the developmental theory examines transition processes utilizing age-linked periods, issue-based resolutions, and domain-specific development stages (Schlossberg, 1981). Similarly, lifespan theory explains transition processes through the lens of individuality, continuity, and change while the transitional framework focuses on change events. The transitional framework is a cognitive appraisal model that focusses on assessing transactional processes between individuals and events (Schlossberg, 1981; Schlossberg et al., 1995). Specifically, the framework focuses on the availability and use of coping resources.

Consequently, transition models allow us to understand the inevitable and sometimes unpredictable processes triggered by change events. Importantly, transition models provide a framework for successfully managing change (Brisson-Banks, 2010). According to Janusz \& Walkiewicz (2018), transition models provide a sequence of successive phases for resolving disruptive life experiences. This paper examined transition models developed by Bridges (2003), Schlossberg et al. (1995), Nicholson (1990), Bussolari and Goodell (2009) and the KIT model was presented as more comprehensive approach to transition management.

\section{Bridges' Model of Transition}

Bridges model categorized transition as a process with an end, neutral, and beginning phase. Bridges (2003) asserted that all transitions start with an ending phase and the end of every transition initiated a new beginning. The ending phase of a transition included disengagement, dis-identification, disenchantment, and disorientation.

Disengagement signified a process in which one separates from an old and familiar identity. The process referred to the beginning of an individual's disconnection from familiar systems that guide accustomed roles and behavior (Bridges, 1980). This was followed by disidentification, a process in which an individual began to lose the identity associated with past routines, roles, and relationships (Schlossberg et al., 1995). Disengagement led to disenchantment a period marked by feelings of disappointment at realizing that an old identity was incompatible with a new situation and discarded. Following disenchantment was a period 
of disorientation, characterized as a period in which individuals felt lost and confused about the next steps to take in restoring a sense of order in his or her experiences.

The second phase of Bridges model is the neutral phase. Bridges (1980) characterized the phase as a period where one reflects and develops a new sense of self. The phase was the beginning of a disengagement process that presented opportunities for a new beginning. However, in transitions, the separation and loss of a familiar context represents a threat to the status quo and tends to heighten feelings of anxiety, vacillation, and uncertainty (Brammer \& Abrego, 1981; Schlossberg, 1981). As a result, emotional, cognitive, and behavioral activity increased to restore calm, stability, and predictability.

According to Bridges (1980), vacillation, uncertainty, and feelings of anxiety led to the need for a temporary timeout. Bridges (2003) referred to this temporary time out as a moratorium period or neutral phase. Consequently, the neutral phase referred to a period in which an individual made attempts to restore calm, stability, and predictability. The period provided one with an opportunity to develop and gain insight on ways to accommodate the transformative experience triggered by a change event (Bridges, 2003; Schlossberg et al., 1995).

Similarly, the final phase referred to a new beginning and included the process of realigning inner experiences with the external realities of a new situation (Bridges, 2001). The process included examining one's inner resistance and gaining an understanding of the obstacles that undermine adaptation. According to Bridges, the process included cognitive, emotional, and behavioral realignments. Importantly, making new beginnings required integrating elements of the old self with a new self-identity (Bridges, 2003; Bussolari \& Goodell, 2009). Consequently, the final phase was viewed as a challenging period in which people find meaning and control over their transitional experiences. Most importantly, it was a period in which one accept a new situation and begins to move forward.

In summary, Bridges' (2003) model provided a clear three-phase approach to transitions that applied to both individuals and organizations. The model used an end, neutral, and beginning phase to characterize the process of moving in, though, and out of transitions (Schlossberg et al., 1995). However, frameworks sequential linear framework limits the model's ability to capture unpredictable, non-linear and dynamic nature of the transition process (Bussolari \& Goodell, 2009, Kralik et al., 2006). Additionally, although Bridges' model characterized transitions as a clearly delineated process with a beginning, middle, and end, Schlossberg et al. (1995) argued that transitions were a continuous process of appraisal and assimilation with no clear endpoints between phases.

\section{Schlossberg Model of Transition}

Schlossberg's (1995) model presented an approach to transitions centered on the processes of assessment and taking charge. The model was based on the assumptions that transitions are continually experienced and reactions to transitions depend on the type of transition, context, and impact. Furthermore, Schlossberg et al., (1995) described a transition as a continuous process of assimilation and appraisal with no endpoints.

Consequently, Schlossberg et al. (1995) proposed a 4S model to assess transitions. The major variables assessed include 1) situation, 2) self, 3) support, and 4) strategy. The situation variable referred to circumstances and factors surrounding a transition while the self-variable signified individual characteristics and resources one could access to cope with transitions. Similarly, support referred to the assistance available in aiding an individual in cope with transitions while strategy referred to an individual's plan of action to cope with a transition. 
Specifically, the model appraised an individual's assets and liabilities in coping with transitions. Schlossberg et al. (1995) postulated that more assets made for easier adjustments while liabilities made for challenging transitions.

Importantly, factors associated with the four major variables were assessed. For instance, the factors assessed for every situation included triggers, timing, control, role change, duration, prior experience with similar transitions, concurrent stressors, and appraisal. Similarly, factors assessed of the self-included appraising an individual's personal and demographic characteristics as well as one's psychological resources. Personal and demographic characteristics refer to socioeconomic status, gender, age and stage of life, and ethnicity while psychological resources include ego development, outlook, commitment, and values. Likewise, evaluating the support variables entailed assessing intimate relationships, the family unit, friendship, institutional and organizational support. Finally, appraising strategy included assessing an individual's plan to modify or control the situation by taking direct, inhibitive, information seeking, or intrapsychic action.

In summary, Schlossberg's (1981) model provided a detailed approach to assess variables and factors that influence transitions. The model described how the $4 \mathrm{~S}$ system - Situation, Self, Support, and Strategy - could be utilized to examine an individual's potential assets and liabilities in coping with transitions. However, unlike the Bridges (2003) model that applied to both individuals and organizations, Schlossberg's (1981) model focused on counseling adults in transition. Additionally, while the model offered detailed steps on appraising an individual's assets and liabilities, it did not provide specific interventions or concrete steps on ways to facilitate a successful transition (Hopson, 1981).

Although the model suggested ways to help adults in transition by expanding coping skills, it offered little in the way of coping styles or their impact in navigating through transitions (Hopson, 1981; Schlossberg et al., 1995). Nevertheless, like Bridges (2003), Schlossberg argued that transitions were not a matter of change but an individual's perception of change. In the final analysis, while evaluating assets is critical to the transition process, the ability to utilize assets is important to successful transition outcomes. Consequently, a successful transition depends on an individual's ability and willingness to utilize identified assets. Schlossberg's model did not address ways to facilitate ability or willingness of an individual in using identified assets.

\section{Nicholson Model of Transition}

The Nicholson (1990) model examined causes, trends, and challenges of transitions in occupational settings. Nicholson utilized predictive theory, process model, and a taxonomic approach to explaining adaptation to change. The use of a predictive theoretical approach assessed multiple factors and provided a prognostic outcome to a transition. Specifically, Nicholson's model focused on predicting personal change and role development. Nicholson (1990) asserted that motives of people entering transitions and the differences experienced between new and old roles determined transition outcomes. For instance, a study by Nicholson and West (1988) demonstrated that anxious people before a transition experienced personal change, while those with high growth needs experienced role development. However, Ashforth and Saks (1995) argued that while people's motives influenced transition outcomes, the way people experienced transitions was influenced by other critical factors including social referents.

Importantly, Nicholson's (1990) characterized the process model as non-prescriptive to allow for the assessment of a variety of transition experiences. Consequently, guiding the model were 
principles of recursion, disjunction, and interdependence. While recursion referred to continuous movement, disjunction signified the distinctive division of experiences and events into stages. Similarly, interdependence referred to the influence exerted by each stage in a transition cycle to the next stage. Although Nicholson's model was presented as a systematic non-normative framework to accommodate a wide range of transition experiences, the model was designed and used to assess transition experiences in occupational settings (George, 1993).

Specifically, Nicholson (1990) presented the transition cycle as a practical analytical framework that facilitates adaptation. The cycle consisted of four stages: preparation, encounter, adjustment, and stabilization. Preparation referred to one's state of readiness while encounter denoted the ability to cope with an event or experience. Similarly, adjustment referred to one's ability to regulate self through personal change and role development while stabilization referred to the balance and equilibrium that result in successful adaptation. The stages offered useful ways to optimize readiness (preparation stage), minimize negative emotions (encounter stage), provide a supportive environment to harmonize personal change and role development (adjustment stage), and facilitate opportunities to sustain balance and effective adaptation outcomes (stabilization stage).

Furthermore, Nicholson (1990) proposed a nine-dimensional taxonomy system to capture and profile the transition process. The nine dimensions included speed, amplitude, symmetry, continuity, discretion, complexity, propulsion, facilitation, and significance. Nicholson asserted that speed, size, and shape reflected the amplitude and symmetry of the transition process. Similarly, smoothness characterized continuity and complexity while onset reflected propulsion. Likewise, obstacles characterized discretion and facilitation while direction reflected the significance of the transition process.

Additionally, Nicholson (1990) referred to speed as the rate at which the transition occurs and amplitude as the drastic demands of a transition. Similarly, symmetry referred to the proportion of times spent at each stage while continuity referred to the succession of transition cycles (logical vs. illogical patterns). Discretion referred to the ability to determine events and experiences in stages of the transition cycle while complexity signified the multifaceted nature of a transition. Likewise, propulsion denoted the onset or trigger of a transition cycle while facilitation referred to factors that aid progress through the transition cycle. Lastly, significance referred to a transition's impact on the individual (Nicholson, 1990).

In summary, although Nicholson's model assessed and generated data from multiple variable, the model is highly complex with multiple intersecting variables. Furthermore, while Nicholson (1990) asserted the model's intervention strategies were applicable in a wide range of situations, the complexity of the framework makes the model more theoretical and less practical in a full range of transitional experiences. Moreover, the model primarily focuses on occupational transitions and specifically examines adjustments of personal and role development in work-related settings (Ashforth \& Saks, 1995).

Additionally, the model does not address emotional variables associated with the transition process. Kidd (1998) argued that Nicholson's model did not accommodate the role of emotion and cognition in their transition processes. For instance, Nicholson did not account for the impact of positive emotions on personal change or role development transitions outcomes. According to, Fish, Bhanugopan, and Gogin (2008) the model did not examine the role of personal control and coping strategies during transitions. Furthermore, Nicholson's model did not account for cultural factors that influence assimilation and work role transitions. It is 
argued that social and cultural factors need to be taken into account when assessing transitions (Fish et al., 2008; Rossi, 2018).

\section{Bussolari and Goodell Model of Transition}

Bussolari and Goodell (2009) proposed a transition model based on the chaos theoretical framework. The model was centered on the notion that transitions are disorderly, unpredictable, but manageable processes (Bengston \& Allen, 2009). While most change is orderly, uncertainty reduction is a common goal in most transitional activities (Black, Mendenhall, \& Oddou, 1991). However, Bussolari and Goodell argued that transition models were limited in their ability and scope because they utilized a western medical model to address change. Specifically, they argued that western medical models focused on pathology and failed to address the unpredictable, nonlinear, and disorderly transitional experiences of healthy functioning individuals. Bussolari and Goodell (2009) asserted that the western medical model was a reductionist and linear approach to transitions. Consequently, the western medical approach was inadequate in addressing nonlinear psychological and emotional problems associated with transitional experiences.

In contrast to the western medical models, Bussolari and Goodell (2009) presented a framework that focused on utilizing a positive strength approach to transitions. The model aimed to facilitate healthy emotional growth and adaptation (Bridges, 2001). The frameworkcharacterized transitions as a construction and reconstruction process comprised of adjustment and adaptation activities (Berger \& Luckmann, 1966; Israelashvili, 2012). They defined adjustments as temporary measures taken to alleviate distress and adaptation as a restorative process that facilitates successful transition outcomes. Bussolari and Goodell asserted that adaptation was the primary goal of the chaos model.

In their approach, Bussolari and Goodell (2009) characterized transitions as a process of reconciling inconsistencies of comfortable and uncomfortable realities at a cognitive and emotional level. Consequently, they proposed the use of language as a method of supporting the adaptation process. They argued that human beings are meaning-making creatures and language was critical in constructing personal narratives to reconcile conflicting realities (Berger \& Luckmann, 1966; Rosen \& Kuehlwein, 1996). Specifically, language provided a framework by which individuals organize, interpret, and make meaning of their experiences (Berger \& Luckmann, 1966). In sum, Bussolari and Goodell (2009) viewed new narratives as the basis for adaptive change and language as the appropriate medium for facilitating novel meaning, thoughts, feelings, and behaviors.

Consequently, Bussolari and Goodell (2009) proposed a list of twelve language constructs to help people disengage from disordered psychological and emotional narratives. These constructs included adaptation, adjustment, phase transition, self-organization, butterfly effect, bifurcation, emergency, chaos, complex adaptive systems, attractors (point and strange), fractals and networks. The authors characterized adaptation as the vibrant process of alteration and integration of transitional experiences while defining adjustment as an attempt to cope with transitional experiences. The transition phase was described as an uncomfortable period of not knowing, while self-organization was the emerging period of growth. Furthermore, the butterfly effects were small events and experiences that resulted in significant changes while bifurcation was the emergence of new possibilities.

According to Bussolari and Goodell (2009), emergence resulted from a reconstructed self while chaos was indicative of an imminent and required change. In addition, complex adaptive systems were open structures that facilitated an exchange for growth and adaptation while 
attractors were factors that influenced one's engagement in repetitive maladaptive patterns or novel behaviors toward adaptation. Fractals referred to similar life themes while networks reflected the connections between the individual and support systems. Bussolari and Goodell (2009) asserted that the listed terms could help individuals successfully navigate and manage unpredictable transition processes.

In addition, Bussolari and Goodell (2009) proposed narrative reframing to assist individuals moving through transitions. They argued that negative narratives could be reframed by identifying a person's positive coping narratives of optimism, encouragement, and hopefulness (Freedman \& Combs, 1996). Moreover, cognitive behavioral approaches could integrate and facilitate the individual's new narratives. The approach was predicated on changing one's thoughts, affect, and behavior (Meichenbaum, 1977). Specifically, the cognitive-behavioral approach provided structured experiences that helped stabilize routines during disruptive and disordered transition periods.

Although Bussolari and Goodell's model provided language and terminology applied in narrative reframing, it did not provide a clear structure or systematic process that could be used to manage transitions. Additionally, while chaos was presented as undesirable and negative, chaos in transitions can be an opportunity to restore order and expand one's positive coping skills (Bussolari \& Goodell, 2009). Therefore, chaos is not always undesirable or negative. Furthermore, although people can reframe the new and unknown into opportunities for growth, people in transition tend to cling to old familiar experiences and routines because of the challenge presented by fear of a new and unknown future (Brammer \& Abrego, 1981). Importantly, these situations escalate by the experienced sense of loss and grief (Bridges, 1980). While Bussolari and Goodell provided a list of language construct to help navigate people through transitions, they did not provide concrete steps on how to help people successfully reframe long-standing beliefs and routines in the face of fear, loss, or grief. Moreover, they did not provide a process to demonstrate how painful and ambiguous narratives associated with the loss of old familiar routines integrated into a new narrative.

\section{Kennedy's Integrated Transition (KIT) Model}

In this paper, I propose an integrated model that aims to facilitate a comprehensive approach to transition management. Kennedy's Integrated Transition (KIT) framework is inclusive and incorporates elements of Bridges, Schlossberg, Nicholson, and Bussolari and Goodell models. KIT is established on the idea that transitions are constructive cycles with adjustment and adaptation as main objectives (Bussolari \& Goodell, 2009; Karlik et al., 2006). While adjustments are vital to effective transitions, adaptation is critical to a successful transition (Bridges, 2001). More importantly, a successful transition is realized when "...feelings of distress are replaced with a sense of well-being and mastery of a change event" (Karlik et al. 2006, p. 321). Consequently, transition management is essential for a successful adaptation outcome (Bridges, 2003; Bussolari \& Goodell, 2009).

Specifically, the KIT model is grounded in the constructionist theory (Berger \& Lackmann, 1966; Rosen \& Kuehlwein, 1996). The theory postulates that reality is constructed and maintained by relationships (Rosen \& Kuehlwein, 1996). In other words, everyday reality is socially constructed and maintained by peoples' interactions (Berger \& Lackmann, 1966). Similarly, transitions are managed by constructing new narratives, restructuring old assumptions, and developing new interactions (Bussolari \& Goodell, 2009; Kralik et al., 2006). Consequently, managing the transition process is essential for successful adaptation outcomes (Bridges, 2003). However, understanding the transition cycle is critical to facilitating effective 
transition management. Figure one below illustrates the adjustment and adaptation phases in the KIT cycle.

Figure One: Transitions Cycle

\section{TRANSITION CYCLE}



ADAPTATION

ADJUSTMENT



The adjustment phase is comprised of a deconstruction process while adaptation includes a process of reconstruction. The process of deconstruction encompasses disruption and reorganization while reconstruction is characterized by integration and internalization activities. In this paper, deconstruction and reconstruction are presented as vital processes in facilitating successful adaptation outcomes (Bussolari \& Goodell, 2009; Kralik et al., 2006). In addition, the management of disruption, reorganization, integration, and internalization activities is discussed. 
Figure Two: The KIT Model



\section{Deconstruction}

In the KIT cycle, deconstruction is the first step toward adaptation (Bussolari \& Goodell, 2009; Rosen \& Kuehlwein, 1996). It is the phase in which disruptions are assessed, embedded assumptions evaluated, and routine behaviors unpacked (Freedman \& Combs, 1996; McKenzie \& Monk, 1997). Specifically, deconstruction provides an opportunity to assess and contextualize disruptions (McKenzie \& Monk, 1997). Furthermore, the process facilitates a better understanding of embedded assumptions, narratives, and their limited use in a new context (Freedman \& Combs, 1996). Notably, deconstruction initiates the vital process of identifying assumptions, narratives, and behaviors that need reconstruction. (Bussolari \& Goodell, 2006; Rosen \& Kuehlwein, 1996).

More importantly, deconstruction entails the activity of reorganizing assumptions to bridge inconsistencies between established routines and new expectations (Bridges, 2003; Schlossberg, 1981). Reorganization is an important activity because it facilitates the difficult task of reconciling contradictions of comfortable and uncomfortable experiences (Bussolari \& Goodell, 2009). In sum, deconstruction is the process of unpacking disruptions triggered by a change event and reorganizing activities to facilitate adjustments during transitions.

\section{Disruption.}

In the transition process, the KIT model begins with an assessment of disruptive activities. While most models acknowledge disruption as an important element in the transition process, few prioritize it as a critical starting point in assessing transitions. It is important to note that while every transition is triggered by a change event, every transition begins with disruption to a status quo (Bridges, 1980; Schlossberg et al., 1995). Specifically, disruptions are unsettling interruptions to one's established and familiar sense-making process (Kralik et al., 2006). According to Brammer \& Abrego (1981), they unsettle familiar assumptions and interrupt behavioral expectations. In sum, disruptions interrupt established routines, roles, and relationships (Schlossberg, 1995; Janusz \& Walkiewicz, 2018; Pogoysan, 2016). 
More importantly, disruptions are indicators that signal the need for an adjustment. According to Bridges (1980), disruptions signal the need for corrective measures to restore order, balance, and function in a changing world. In the KIT model, disruptions provide the initial signal for corrective action aimed at eliminating interruptions and restoring functions of familiar assumptions and behaviors. Corrective measures include making adjustments to facilitate the development of new assumptions or behaviors (Kralik et al., 2006; Schlossberg et al., 1995). Specifically, transitional adjustments vary based on the intensity of a disruption (Schlossberg et al., 1995). However, few models address or include the varying intensity levels of disruption as presented in the KIT model. Consequently, the KIT model is characterized by levels of low, moderate, and high intensity disruptions.

Low Intensity Disruption (LID). A low intensity disruption (LID) causes little interruption in one's assumptive world and minimal inconvenience in routines, roles, and relationships. Consequently, adjusting to LID is less challenging and requires marginal coping resources. Importantly, LID does not demand significant cognitive, affective, or behavioral adjustments. For example, although waking up at dawn for a favorite yoga class may interrupt one's sleep cycle, it causes minimal disruption in one's assumptive and behavioral world. Therefore, cognitive, affective, and behavioral adjustments demanded by LID compared to those demanded by moderate intensity disruptions is marginal.

Moderate Intensity Disruption (MID). Adjusting to moderate intensity disruption (MID) is more challenging compared to low intensity disruption. An interruption caused by MID is unsettling to an individual's assumptive and behavioral world. It considerably influences one's routines, roles, and relationships. More importantly, MID requires substantial coping resources and demands considerable cognitive, affect, and behavioral adjustments. An example of a moderate intensity disruption is a student leaving home for the first time to attend college as a freshman in a distant state. The disruption can be exacerbated if a student is the first person in his or her family to attend college.

High Intensity Disruption (HID). High intensity disruptions are severe and cause acute interruption in one's routines, roles, and relationships. They are significantly unsettling and challenging to an individual's assumptive and behavioral world. Importantly, HID requires significant coping resources and demands major cognitive, affect, and behavioral adjustments. An example of HID is the loss of a loved one or the diagnosis of a loved one with a terminal illness. The categorization of disruptions by intensity levels are a key feature of the KIT model.

However, it is important to note that disruptions caused by change events are fluid. Therefore, a change event can trigger new or multiple concurrent disruptions. For instance, choosing to attend college across the country for a great scholarship opportunity may stabilize financial disruptions but destabilize one's social support system ushering in a new set of disruptions. Likewise, an individual may experience multiple and concurrent disruptions. For instance, having a newborn child and getting laid off are examples of change events that could trigger multiple and concurrent disruptions. Similarly, moving across the country to attend college can be a cultural dislocation that simultaneously triggers social, academic, and emotional disruptions. In summation, while disruptions are categorized and presented as low, moderate, and high intensity in this paper, the way they are experienced by individuals varies.

\section{Reorganization}

In the KIT model, reorganization is the activity of reassembling old assumptions and behaviors to meet the demands of a change event. The activities lay a foundation for integrating and facilitating readiness for the reconstruction process (Bridges, 1980; Nicholson, 1990). The 
objective of reorganization is to minimize distress and facilitate acceptance of a new situation (Moos \& Tsu, 1976; Schlossberg, 1981). During reorganization, a variety of options are explored and tentative modifications made to minimize the impact of a disruptive event (Brammer and Abrego, 1981). Importantly, reorganization signals a period of psychological realignment and the beginning of cognitive, affect and behavioral restructuring (Bridges, 2003). Specifically, reorganization activities include reconstituting established assumptions and worldviews in preparation for reconstruction (Parkes, 1971; Schlossberg, 1981). As asserted by Zittoun (2006), actualization of a transition occurs when experiences are reorganized within a set of new constraints.

\section{Reconstruction}

In the KIT model, reconstruction is the beginning of a transformative process (Rosen \& Kuehlwein, 1996). It entails integrating and internalizing new assumptions and behaviors. Reconstruction provides an opportunity for individuals to restructure their narratives and make sense of their life experiences (Bussolari and Goodell, 2009). Specifically, the process facilitates a restoration of positive narratives and reestablishment of a valued self-identity (Bridges, 2001; Kralik et al., 2005). During reconstruction, reassembled assumptions and behaviors are integrated and internalized to meet the expectations of a new reality. Therefore, reconstruction integrates new perspectives and internalizes new ways of thinking, feeling, and acting (Zittoun, 2006).

\section{Integration}

Integration is the activity of merging elements of new and old identities together (Bridges, 1980). Mainly, integration involves incorporating reassembled assumptions and adjusting behaviors into new routines. Specifically, the activities of integration consist of incorporating assumptions and behaviors that produce positive outcomes while discarding those that produce negative experiences (Brammer \& Abrego, 1981). Consequently, integration is the period in which an individual's disparate assumptions and experiences are brought into a congruent, cohesive, and consistent biographical framework (Berger \& Lackmann, 1966). Specifically, integration is the beginning of the reconstruction process. It is a period in which assumptions are reframed and the values demanded by a new situation are adapted (Brammer \& Abrego, 1981). More importantly, integration is the activity of reconciling and merging new and old experiences in preparation for internalization (Zittoun, 2006).

\section{Internalization}

Internalization is a period in which the realities of a new situation are absorbed and accepted into consciousness (Berger \& Lackman, 1966). Consequently, internalization includes introjection and assimilation processes. Introjection entails taking in new values while assimilation refers to the process of accepting new values as an integral part of the core sense of self (Deci, Eghrari, Patrick, \& Leone, 1994). Internalization occurs when an intrinsically rewarding assumption or behavior is adopted (Kelman, 1958). Ordinarily, assumptions and behavior that are congruent with one's value system are adopted.

Therefore, the process of internalization involves synchronizing cognitive, affect, and behavioral actions with the demands of a change event. Internalization is completed when newly acquired assumptions and behaviors are accepted as a way of life and new routines, roles, and relationships are habituated (Bussolari \& Goodell, 2009; Schlossberg, 1995). Specifically, internalization is a transformative and adaptive process that restores equilibrium and a lasting sense of stability (Bussolari \& Goodell, 2009). It denotes the end of a transition period and a restored stable valued self-identity (Schlossberg, 1981). It is the period in which 
"...feelings of distress are replaced with a sense of well-being and mastery of a change event" (Karlik et al. 2006, p. 321).

\section{SUMMARY AND IMPLICATION OF THE KIT MODEL}

The KIT model presents a coherent and comprehensive approach to transitions. It addresses limitations in past transition models while incorporating key transition elements. For instance, unlike the sequential 3 phase linear framework proposed by Bridges, the KIT model presents a 2-phase cyclical framework of adjustment and adaption. Importantly, the KIT model accommodates considerable variations of transition experiences. The model's framework captures unpredictable, non-linear, and fluid experiences of the transition process (Bussolari \& Goodell, 2009, Kralik et al., 2006; Nicholson, 1990). This is in contrast to Schlossberg's model aimed to counsel adults in transition, or Nicholson's model focused at occupational transitional experiences, (Schlossberg et al., 1995; Nicholson, 1990).

In addition, the KIT model appraises and manages normative or unique transition experiences. For instance, while the model can appraise routine low intensity disruptions, it can manage challenging high intensity disruptive life events such as the loss of a loved family member. More importantly, the processes and activities assessed in the KIT model form the basis of every transition experience. For instance, every change event (normative or unique) disrupts familiar routine, roles, and relationships. Consequently, disruptions trigger the need for adjustment and reorganization. These activities alleviate distress and meet demands of the change event. Subsequently, a reconstruction process that includes integration and internalization activities are triggered to help merge reassembled assumptions and introject into consciousness new behaviors. These processes and activities are in every transition experience (Bridges, 2003; Bussolari \& Goodell, 2009, Kralik et al., 2006; Nicholson, 1990; Schlossberg et al., 1995).

The KIT model provides a framework with a clear structure and systematic process that can guide one's navigation through the unstructured and chaotic nature of a transition (Bussolari \& Goodell, 2006). The framework provides an understanding of transition processes and activities triggered by change events. In contrast to past models, KIT accommodates considerable variation in transition experiences. The model supports the assertion, "it isn't the changes that do you in, it's the transitions." (Bridges, 2003, p. 3). More importantly, it affirms that transition management is essential to successful adaptation outcomes.

However, while KIT presents a framework that provides a more comprehensive approach to transition management, the model does not unilaterally account for successful transition outcomes. In other words, the KIT model is one of many variables that contribute to successful transition outcomes. For instance, while the KIT model provides a framework making transition processes understood, effective coping behavior and social support systems are essential for successful transition outcomes. However, the model provides a framework within which a coping strategy or behavior can be assessed, developed, and effectively implemented. Importantly, the model provides a framework to guide, manage, and leverage adaptive coping processes for successful transition outcomes.

Although discussing the coping process is beyond the scope of this paper, it is important to note that managing a coping process is critical to successful transition outcome. Briefly, coping is an ongoing cognitive, emotional, and behavioral effort used by an individual to manage specific external and/or internal demands of a stressful and disruptive situation (Lazarus, 1993; Taylor \& Stanton, 2007). Importantly, an adaptive coping process includes the appraisal of resources and an implementation of a coping strategy. Specifically, coping resources are a 
set of skills and support systems that an individual can readily access to manage demands of a distressing or disruptive situation. Similarly, a coping strategy is a deliberate plan designed to overcome, minimize, or tolerate stress or conflict triggered by a disruptive and distressing situation. In sum, managing the coping process is important in facilitating successful transition outcomes. However, coping successfully requires mobilizing resources and implementing an effective strategy.

In conclusion, there are multiple factors that facilitate adaptation. The KIT model provides a framework in which managing transition processes can result in successful outcomes. However, it is important to note that effective coping processes and access to support systems are essential to successful transition outcomes. Although specific details on how to assess, develop, or facilitate effective coping processes are beyond the scope of this paper, the KIT model provides a framework to manage effective coping. Specifically, the model provides a framework to examine and assess variations of transitional experiences. Importantly, it provides a framework in which coping can be assessed and strategies leveraged to facilitate successful transition outcomes. However, further empirical research data is needed to assess and evaluate the KIT model's effectiveness in facilitating coping processes to enable successful transition outcomes.

\section{References}

Ashforth, B. E., \& Saks, A. M. (1995). Work-role transitions: A longitudinal examination of the Nicholson model. Journal of Occupational and Organizational Psychology, 68(2), 157-175.

Black, J. S., Mendenhall, M., \& Oddou, G. (1991). Toward a comprehensive model of international adjustment: An integration of multiple theoretical perspectives. Academy of Management Review, 16(2), 291-317.

Bengtson, V. L., \& Allen, K. R. (2009). The life course perspective applied to families over time. Sourcebook of Family Theories and Methods, 469-504.

Berger, P. L., \& Luckmann, T. (1990). The social construction of reality: a treatise in the sociology of knowledge. New York: Anchor Books.

Brammer, L. M., \& Abrego, P. J. (1981). Intervention strategies for coping with transitions. The Counseling Psychologist, 9(2), 19-36.

Bridges, W. (2003). Managing transitions: making the most of change: 2nd ed. Cambridge, MA: Perseus Books.

Bridges, W. (2001). The way of transition: Embracing life's most difficult moments. Cambridge, MA: Perseus Pub.

Bridges, W. (1980). Transitions: making sense of life's changes. Cambridge, MA: Perseus Books.

Brisson-Banks, C. V. (2010). Managing change and transitions: a comparison of different models and their commonalities. Library Management, 31(4/5), 241-252.

Bussolari, C. J., \& Goodell, J. A. (2009). Chaos theory as a model for life transitions counseling: Nonlinear dynamics and life's changes. Journal of Counseling \& Development, 87(1), 98-107.

Deci, E. L., Eghrari, H., Patrick, B. C., \& Leone, D. R. (1994). Facilitating internalization: The self-determination theory perspective. Journal of Personality, 62(1), 119-142.

Fish, A., Bhanugopan, R., \& Cogin, J. (2008). Value orientations as predictors of cultural and business impact: Individual suitability for cross-border assignments. Cross Cultural Management: An International Journal, 15(1), 30-48

Freedman, J., \& Combs, G. (1996). Narrative therapy: The social construction of preferred realities. WW Norton \& Company.

Hopson, B. (1981). Response to the papers by Schlossberg, Brammer and Abrego. The Counseling Psychologist, 9(2), 36-39.

George, L. K. (1993). Sociological perspectives on life transitions. Annual Review of Sociology, 19(1), 353-373. Golan, N. (1983). Passing through transitions. Simon and Schuster. 
Israelashvili, M. (2012). Should adjustment disorder be conceptualized as transitional disorder? In pursuit of adjustment disorders definition. Journal of Mental Health, 21(6), 579-588.

Janusz, B. \& Walkiewicz, M. J Adult Dev (2018). https://doi.org/10.1007/s10804-018-9285-1

Kelman, H. C. (1958). Compliance, identification, and internalization three processes of attitude change. Journal of Conflict Resolution, 2(1), 51-60.

Kidd, J. M. (1998). Emotion: An absent presence in career theory. Journal of Vocational Behavior, 52(3), 275288.

Kralik, D., Visentin, K., \& Van Loon, A. (2006). Transition: a literature review. Journal of Advanced Nursing, 55(3), 320-329.

Lazarus, R. S. (1993). Coping theory and research: Past, present, and future. Fifty years of the research and theory of RS Lazarus: An analysis of historical and perennial issues, 366-388.

Levine, S. V. (1976). Draft dodgers: Coping with stress, adapting to exile. American Journal of Orthopsychiatry, 42(3), 431.

Meleis, A. (2010). Transitions theory middle-range and situation-specific theories in nursing research and practice. New York: Springer publishing company.

Meleis, A. (2015) Nursing theories and nursing practice New York: Springer publishing company.

Meichenbaum, D. (1977). A cognitive-behavior modification approach to assessment. In Cognitive-Behavior Modification (pp. 229-259). Springer, Boston, MA.

Miller, T. W. (2010). Handbook of stressful transitions across the lifespan. New York, NY: Springer New York.

Monk, G. E., Winslade, J. E., Crocket, K. E., \& Epston, D. E. (1997). Narrative therapy in practice: The archaeology of hope. Jossey-Bass.

Moos, R. H., \& Tsu, V. (1976). Human competence and coping: An overview. Human adaptation: Coping with life crises, 3-16.

Nicholson, N. (1990). The transition cycle: Causes, outcomes, processes and forms. On the move: The Psychology of Change and Transition, 83-108.

Nicholson, N., \& West, M. (1988). Managerial job change: Men and women in transition. Cambridge University Press.

Oatley, K. (1990). Role transitions and the emotional structure of everyday life. On the move: The Psychology of Change and Transition, 67-81

Parkes, C. M. (1971). Psycho-social transitions: a field for study. Social Science \& Medicine, 5(2), 101-115.

Pogosyan, M. (2016, March 07). Understanding Transition Stress. Retrieved May 30, 2018, from https://www.psychologytoday.com/us/blog/between-cultures/201603/understanding-transition-stress

Rosen, H., \& Kuehlwein, K. T. (1996). Constructing realities: Meaning-making perspectives for psychotherapists. San Francisco, CA: Jossey-Bass Publishers.

Rossi, A. (2018). Gender and the life course. Routledge.

Schlossberg, N. K. (1981). A model for analyzing human adaptation to transition. The Counseling Psychologist, 9(2), 2-18.

Schlossberg, N. K. (1995). Counseling adults in transition: Linking practice with theory. Springer Publishing Company.

Taylor, S. E., \& Stanton, A. L. (2007). Coping resources, coping processes, and mental health. Annual Review of Clinical Psychology, 3, 377-401.

Zittoun, T. (2006). Transitions: development through symbolic resources. Greenwich, CT: Information Age Pub. 